\title{
ARTHROPLASTY OF THE KNEE BY THE WALLDIUS PROSTHESIS
}

\author{
G. Blundell Jones, Exeter, England \\ From the Princess Elizabeth Orthopaedic Hospital, Exeter
}

This paper presents a review of forty-five arthroplasties of the knee performed between 1961 and 1967, using the prosthesis designed in Sweden by Börge Walldius. The results presented are the combined experience of four surgeons: Mr F. C. Durbin, eleven knees; Mr R. Merryweather, thirteen knees; Mr R. S. Ling, four knees; and myself, seventeen knees. There were forty-five arthroplasties in forty-two patients, three patients having had operations on both knees. The average age at operation was fifty-nine, the youngest patient being thirtyfive and the oldest seventy-eight. The operation was performed for rheumatoid arthritis in thirty-seven patients and for osteoarthritis in five. Seven patients already had arthrodesis of the opposite knee. The number of operations each year was as follows: 1961 four; 1962 seven; 1963 three; 1964 six; 1965 nine; 1966 eleven; 1967 five.

\section{THE PROSTHESIS}

The prosthesis is now made entirely in Vitallium (Fig. 1). The range of movement of the hinge is from 5 degrees hyperextension to 90 degrees of flexion. The two halves of the prosthesis are united by a roller bearing held in place by a locking screw. The thickness of the prosthesis is three centimetres, which enables arthrodesis of the knee to be carried out if the result is unsatisfactory. The femoral half of the prosthesis carries an intramedullary nail eight centimetres long, and a flange in front provides an articular surface for the patella. The tibial component carries an intramedullary nail of the same length, and a posterior flange which fixes in the cancellous bone. The two-point fixation effectively prevents the prosthesis from twisting and sliding, and weight is borne on a flat surface of femur and tibia of area twenty-one square centimetres $\left(6 \times 3 \frac{1}{2}\right.$ centimetres). Thus the prosthesis does not rely on intramedullary fixation and, although the nails are made longer (ten centimetres) in the later models, some surgeons prefer to have them shortened to the original length (eight centimetres) for ease of insertion. Cement has not been used and is felt to be undesirable not only because of the nature of the broad medullary cavity but because subsequent arthrodesis would be difficult should the prosthesis have to be removed. A further reason is that we like the prosthesis to sink in a little so that bone embraces it.

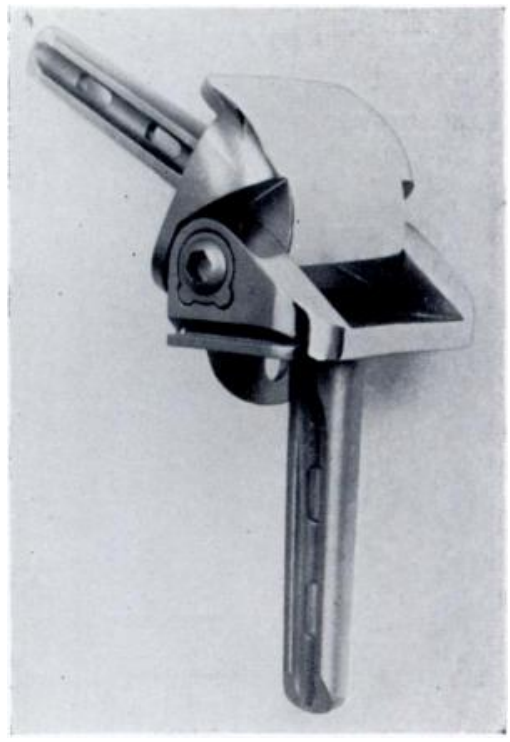

Fig. 1

The Vitallium Walldius knee prosthesis.

\section{TECHNIQUE OF OPERATION}

Walldius (1960) recommended a U-incision extending from the medial to the lateral ligament, reflecting upwards the patellar tendon with its bony insertion and expansion, so exposing the joint surfaces without disturbing the patella or the synovium. We have noted delay in skin edge healing in four cases. A median parapatellar incision has been used in five patients, but the disturbance to the suprapatellar pouch seems to cause greater difficulty in regaining movement. The articular surfaces are cut away by a saw to give sufficient clearance 
for the prosthesis as judged by a three-centimetre measuring block. A flexion deformity of as much as $\mathbf{3 0}$ or $\mathbf{4 0}$ degrees was corrected in nine cases. In two cases with greater deformity wedging plasters were used first to obtain correction. It is also possible to correct quite severe valgus and varus deformities. It is necessary to remove the posterior part of the femoral condyles to prevent impingement. Failure to do so results in limitation of flexion. In ore case new bone formation occurred at this site and prevented flexion beyond 60 degrees.

After operation the leg is immobilised in plaster from groin to toes, and weight-bearing is deferred for two to three weeks until the soft tissues are soundly healed. Thereafter a groin-toankle plaster is applied and weight-bearing is encouraged. The plaster is split at four to six weeks after operation to allow exercise of the knee. Splintage is discontinued in most cases at eight weeks.

\section{SELECTION OF PATIENTS FOR OPERATION}

Like Walldius we were cautious in the beginning and used the operation only for patients who had become unable to walk or almost so, but as the results were encouraging we have since performed the operation a little earlier. All the patients except five were suffering from rheumatoid arthritis, with other disabilities besides arthritis of the knee, so that the demand on the operated joint was much reduced. We do not believe that this operation should be used in any patient who will be capable of vigorous activity but rather as a salvage procedure for the relief of pain and maintenance of limited walking capacity, usually with a stick, in older patients.

\section{RESULTS}

Range of movement-In general, the better the range of movement before operation the sooner it is regained subsequently. Knees with a range less than 20 degrees before operation have remained permanently very restricted, but a range of as little as $\mathbf{4 5}$ degrees is not a contraindication to arthroplasty, and several such patients obtained the full range of the prosthesis.

TABLE I

Knee Movement Regained

\begin{tabular}{|c|c|}
\hline Number of cases & $\begin{array}{c}\text { Maximum flexion } \\
\text { regained (degrees) }\end{array}$ \\
\hline 13 & 90 \\
\hline 10 & 80 \\
\hline 10 & 70 \\
\hline 7 & 60 \\
\hline 1 & 50 \\
\hline 2 & 30 \\
\hline 2 & less than 30 \\
\hline
\end{tabular}

TABLE II

The Fate of the Prostheses

\begin{tabular}{|c|cc|}
\hline $\begin{array}{c}\text { Time since } \\
\text { operation } \\
\text { (years) }\end{array}$ & $\begin{array}{c}\text { Total prostheses } \\
\text { inserted } \\
\text { each year }\end{array}$ & $\begin{array}{c}\text { Number of } \\
\text { prostheses still } \\
\text { satisfactory }\end{array}$ \\
\hline 6 & 4 & 1 \\
\hline 5 & 7 & 3 \\
\hline 4 & 3 & 2 \\
\hline 3 & 6 & 5 \\
\hline 2 & 9 & 8 \\
\hline 1 & 11 & 10 \\
\hline$\frac{1}{2}$ & 5 & 5 \\
\hline
\end{tabular}

Most patients regained the maximum range by the end of six months, but one in three required manipulation under anaesthesia. It is sometimes worth while repeating the manipulation if satisfactory movement is not regained the first time. However, a few patients improve slowly; one of the first cases seemed disappointing, with only 30 degrees of flexion at the end of a year, but the patient regained flexion of 60 degrees at fifteen months and 90 degrees at two years. The range of knee movement regained in forty-five cases is shown in Table I.

Relief of pain-None of the patients complained of disabling pain after operation, though a few suffered aching. This tends to improve as synovial reaction subsides. Even if much 

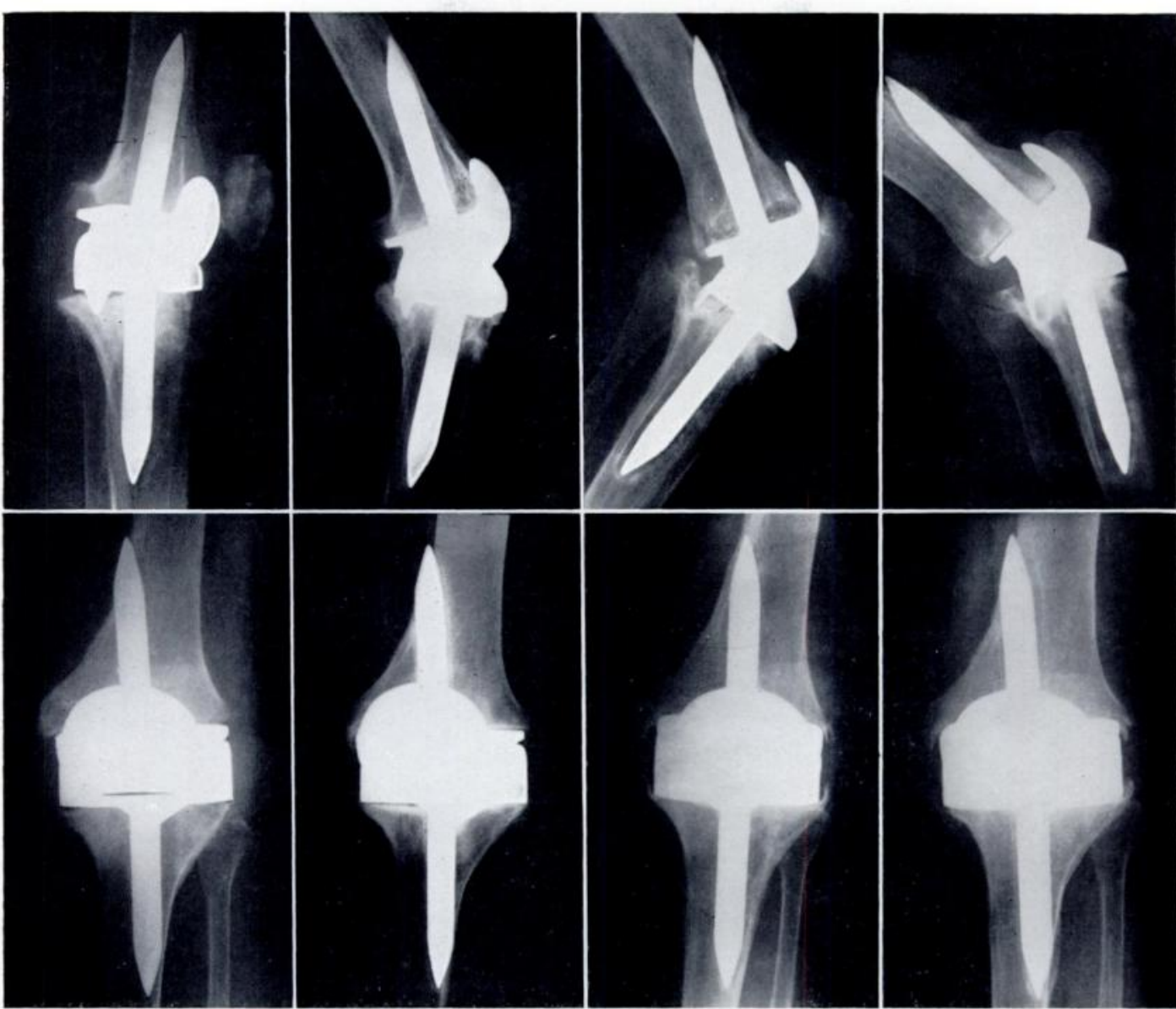

FIG. 2

Serial radiographs immediately after insertion of prosthesis, and at eleven, fifty-four and seventy-three months. There is evidence of loosening of the prosthesis in the tibia, but clinically the result was satisfactory.

synovial thickening is present, once the prosthesis has been inserted it settles down and most of the swelling disappears within six months. Synovectomy has not been done at the time of arthroplasty, but two patients had had synovectomy a few years previously.

Gait-Most patients walk with a stick, carrying the knee rather stiffly but flexing well for stairs. The tendency to walk stiff-legged seems to be due to lack of joint sensation and can be overcome when the patient thinks about it. This stiffness does not appear to affect the knees so much in bilateral cases. Absence of rotation has not worried the type of patient with limited walking capacity whom we chose for the operation.

Failures and complications-1) Infection occurred in three knees, requiring removal of the prosthesis at seven months, fourteen months and two years after operation. Arthrodesis was obtained in two out of the three. One of these patients developed acute septic arthritis after a year of satisfactory function, with 90 degrees of flexion and no immediate post-operative complication. 2) Four other patients had delayed skin healing but after further immobilisation in plaster, and split-skin grafting in one instance, all healed and remained satisfactory afterwards. 3) In no case was there serious failure of the prosthesis, and the short medullary nails have not broken. In one patient the locking screw of the bearing came loose but the joint still functions well. 4) Common peroneal palsy occurred in two patients after operation but cleared up spontaneously within a few months. The fate of the prostheses is shown in Table II.

VOL. 50 B, No. 3, AUGUST 1968 


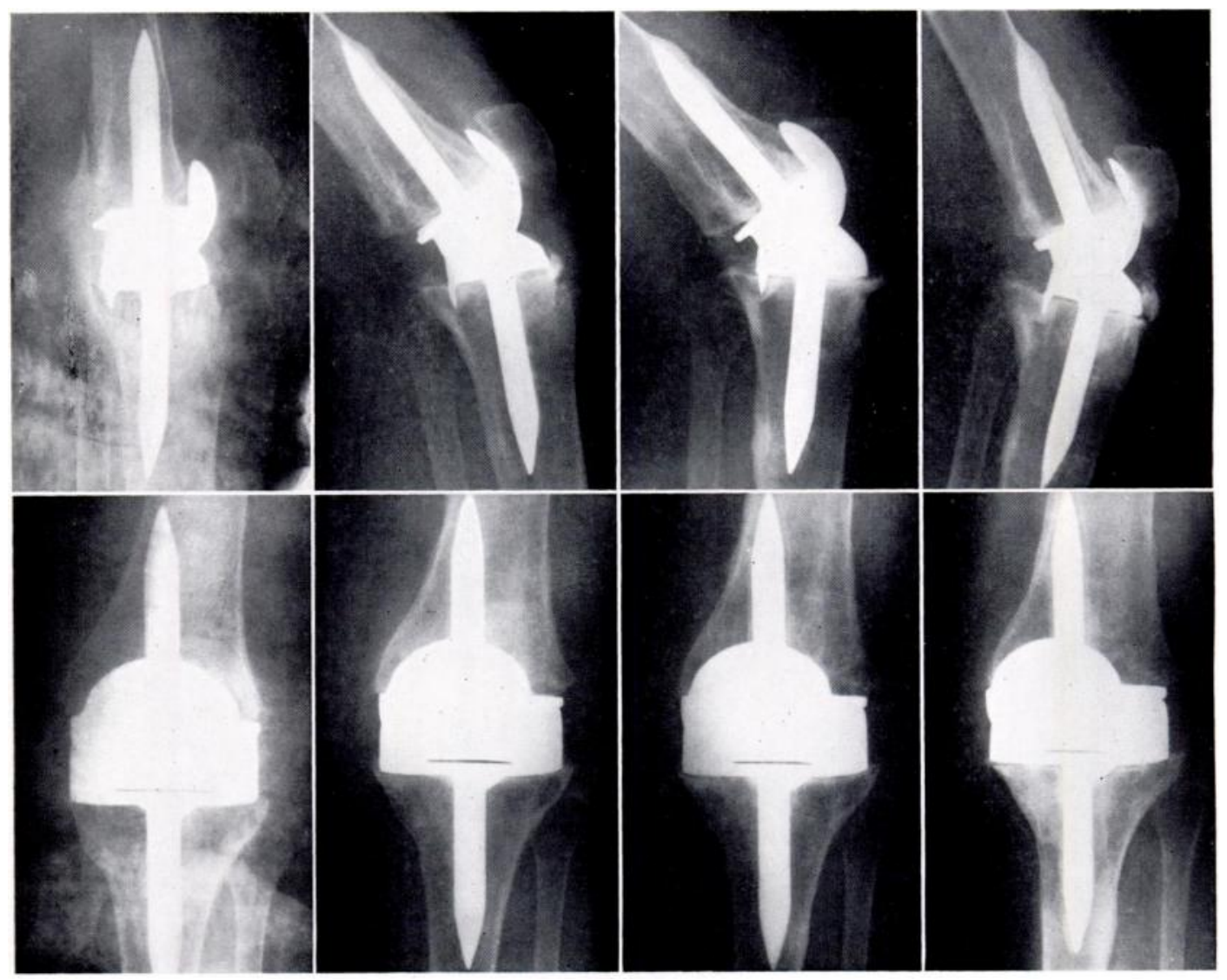

Fig. 3

Radiographs showing successive changes in a patient with rheumatoid arthritis after operation, and at eight, nineteen and fifty-one months. Movement is occurring about the prosthesis.

Five patients died from other causes between six months and five years after operation. The remainder were all available for follow-up. Two patients had excision of the patella some time after the prosthesis was inserted, in the hope of increasing the range of movement. A man of thirty-five who had had rheumatoid arthritis for twenty years had only a few degrees of movement in the knee when operated upon and the patella was fixed. Six months after insertion of the prosthesis patellectomy was done, increasing the movement from 0 to 20 degrees to 5 to 55 degrees. A woman of sixty-five who had had fibrous ankylosis of both knees for many years gained no useful movement seven months after insertion of the prosthesis and was not improved by excision of the patella.

Late complications-Atrophic changes in the bone often render it very soft, and the prosthesis can sometimes be pushed in manually. Occasionally the intramedullary nail penetrated the femoral or tibial cortex but this did not seem to cause trouble. Even if the bone is very soft at the time of operation, it appears to harden considerably during the time of weight-bearing in plaster, and subsequent radiographs show that it beds firmly into position, with sclerosis in the bone over the weight-bearing surface. Lips of new bone form which embrace the prosthesis and further prevent its movement. Figure 2 shows the present condition in one of the first cases, in which the knee retains 90 degrees of movement and remains entirely satisfactory after six years. Radiographs suggest that there is some movement about the nail in the tibia but this does not seem to cause any symptoms. Similar changes have been noted in six other cases. 

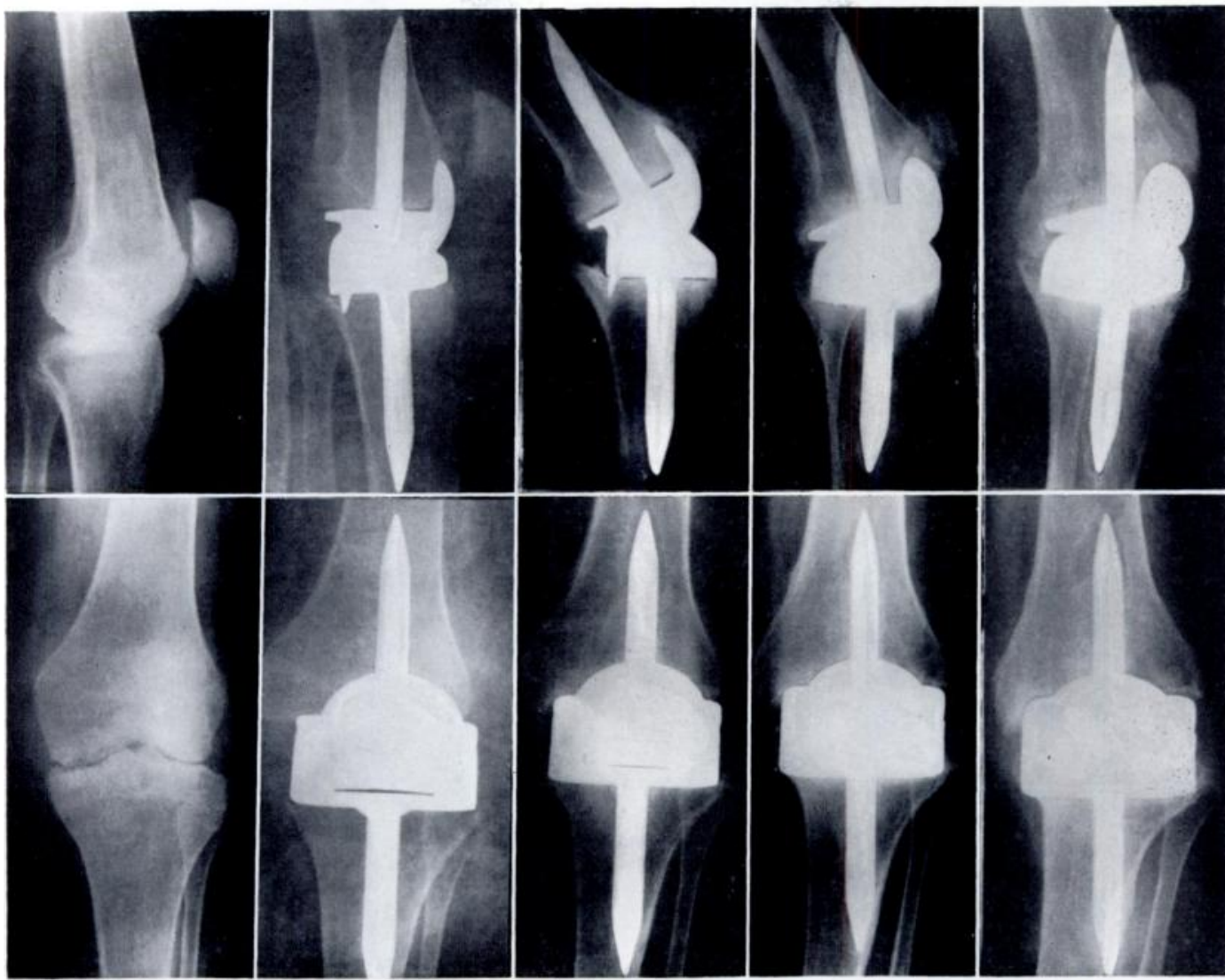

Fig. 4

Successive radiographs before operation, immediately after operation, and at nine, twenty-seven and fifty-one months showing increasing varus deformity. Function, initially good, has deteriorated.

Only one prosthesis became seriously loose with recurrence of severe disability (Fig. 3). This patient had rheumatoid arthritis which has become active again in the past year. She has now become so disabled that it was thought to be unwise to reinsert the prosthesis. The radiograph shows the movement particularly in the tibia, with some periosteal new bone formation at the site of impingement of the end of the nail.

One patient developed severe varus deformity of the knee during the four and a half years after the prosthesis was inserted (Fig. 4). Initial function was exceptionally good. Varus deformity is now 15 degrees while bearing weight and 10 degrees while recumbent. The range of movement, which was initially 10 to 80 degrees, is now reduced to 10 to 40 degrees. This patient has been advised to have the prosthesis removed and reinserted.

\section{RESULTS BY YEAR OF OPERATION}

1961 (four knees) - One survivor has a stable knee after six and a half years, with a range 0 to 80 degrees. This patient also has a cup arthroplasty in the opposite hip, with excellent function twelve years after operation. One patient developed late sepsis one year after operation after having had 90 degrees of movement. The prosthesis was removed and arthrodesis obtained by the Charnley method. Two patients died of other causes two years after operation, with good function and 90 degrees of flexion.

1962 (seven knees) - Two prostheses required removal for sepsis. Of the other five knees, three are good, with 70 degrees or more of flexion. Two, very stiff before operation, later had 
excision of the patella; one of these remained stiff but the other has a useful knee with a range 5 to 55 degrees.

1963 (three knees) - One patient died from unrelated causes in 1965 having enjoyed good function with 90 degrees of flexion. Two others remain satisfactory, also with 90 degrees of flexion.

1964 (six knees)-Five have more than 70 degrees of movement and good function. The other had a very stiff knee before operation with a 30 degree flexion deformity and both hips involved. The range obtained was 0 to 35 degrees only.

1965 (nine knees) - One patient died from unrelated causes six months after operation with a range of 10 to 70 degrees. Six remaining knees have now 70 degrees or better of flexion and the other two 60 degrees of flexion. All are free of pain and functioning well.

1966 (eleven knees) - One patient died from unrelated causes. One had delayed wound healing but healed soundly with antibiotics. Seven have at least 70 degrees of movement. Three have 60 degrees of flexion. All function well.

1967 (five knees)-All those recorded were done more than six months ago. Four have at least 70 degrees of flexion, and one has 60 degrees.

\section{CONCLUSIONS}

We believe that the Walldius prosthesis is the best design at present available and report no structural failure in six years' experience. The massive foreign body is tolerated well by the bone, which hardens around it and embraces it with new bone, improving its stability. If removal becomes necessary for any reason, arthrodesis of the knee can be carried out. We wish to stress that this is a salvage operation whose application should be limited to patients who are not going to make heavy demands on the reconstructed joint. With this qualification the results have been most gratifying and have enabled many patients to continue walking who would otherwise have become chairborne.

\section{SUMMARY}

1. Forty-five arthroplasties of the knee with the Walldius prosthesis are reported in forty-two patients, thirty-seven with rheumatoid arthritis and five with osteoarthritis.

2. There has been no structural failure of the prosthesis.

3. Sepsis required removal of the prosthesis in three cases, but arthrodesis was obtained in two of the three.

4. Serious loosening of the prosthesis occurred in one case only. Varus deformity occurred in one patient.

5. Radiological evidence of some movement of the prosthesis was present in six other cases but this did not seem to affect function.

6. It is concluded that this operation has a place in the treatment of the more seriously disabled patient, but should not be used when heavy demands are likely to be made on the joint.

I am indebted to my colleagues, Mr F. C. Durbin and Mr R. S. M. Ling of Exeter and Mr R. Merryweather of Gloucester, for allowing me to include their cases.

\section{REFERENCES}

Walldius, B. (1960): Arthroplasty of the Knee Using an Endoprosthesis. Acta Orthopaedica Scandinarica, 30, 137.

Walldius, B. (1964): Arthroplasty of the Knee Using an Endoprosthesis. Société Internationale de Chirurgie Orthopédique et de Traumatologie. Neuvième Congrès Vienne, 1-7 Septembre 1963, p. 214. Bruxelles: Imprimerie des Sciences.

Walldius, B. (1968): Prosthetic Replacement of the Knee Joint. Journal of Bone and Joint Surgery, 50-B, 221. 